\title{
ADAPTATION INVESTMENTS FOR TRANSPORT RESILIENCE: TRENDS AND RECOMMENDATIONS
}

\author{
MARIA PREGNOLATO ${ }^{1} \&$ DAVID A. DAWSON ${ }^{2}$ \\ ${ }^{1}$ School of Engineering, Newcastle University, Newcastle (UK). \\ ${ }^{2}$ School of Civil Engineering, University of Leeds, Leeds (UK).
}

\begin{abstract}
Climate change, extreme weather and flooding threaten to increase damage and disruption to our transport networks and the services that they provide. There is increased need for adaptation to maintain current asset conditions and services, and a strategic requirement to prioritise such investments in adaptation to reduce future risks. Physical network risks will not be evenly distributed across nations (e.g. due to geographical and climate change patterns), and some regions will require more investment and adaptive interventions than others to maintain services due their vulnerability to natural hazards. Comparatively, the distribution of investment for transport infrastructure does not have a uniform spatial distribution, and can favour schemes that reduce congestion on networks with high demand without considering the actual risk of being impacted. These two issues, if unchallenged, will present an unfavourable future for areas with high network risks and low transport demand that will widen spatial inequality or resilience, mobility and potential for economic growth. This study advances a methodological framework to analyse the spatial distribution of flood risk on UK road and rail networks in the light of potential bias of regional investment. Using GIS mapping, network data and risk analysis, regional futures are categorised and discussed. There is a clear North/South divide in transport networks at risk from potential coastal and fluvial flooding, with southern regions having 10-30\% of their network situated in known flood risk areas. Investment in transport infrastructure is also disproportionately favoured towards regions with high transport demand, and peripheral regional such as Wales and the South West are at risk from increase disparity from high flood risk networks and a low potential for investment. The study provides preliminary evidence for the need to consider assessment approaches for long-term investment in resilience, drawing recommendations for future research.

Keywords: adaptation, flood, risk, investment, network, rail, resilience, road, transport.
\end{abstract}

\section{INTRODUCTION}

Climate change is now almost unanimously considered as one of the key global challenges to threaten current and future generations. There is a growing consensus that the last few decades have observed increases in temperatures, sea-levels and extreme weather events [1]. Coupled with this, growing populations and urbanisation processes have led to the development of contemporary cities and societies that are increasingly reliant on urban infrastructure systems (e.g. transport, water, waste, energy, communication) for their functioning. However, many of these systems were designed and constructed decades ago before the widespread understanding of long-term environmental change, demand trends, and resilience and they are inevitably aging. As a result, temporary and permanent disruption from direct or indirect (through interdependencies) failures may become more common. The long-term resilience of our infrastructure systems and the ability to withstand threats of climate change, extreme weather patterns, and increasing levels of service demand remains a key societal agenda. Transport infrastructure is particularly exposed to climate and weather, and while research interest in the vulnerability and resilience of transport networks is growing [2], the long-term implications on adaptation investment and decision making have yet to be challenged, preferably by interdisciplinary research approaches [3]. As a generalisation, funding for transport

${ }^{1}$ ORCID: https://orcid.org/0000-0002-2630-4497 2 ORCID: http://orcid.org/0000-0003-0796-9618 
infrastructure schemes in developed countries prioritises expanding capacity, reducing congestion and increasing journey times, which is determined to have a greater impact on economic performance by promoting economic growth and productivity [4, 5]. However, current investment in transport infrastructure is not geographically uniform, and is governed by a mix of political, social, and economic factors. In the UK, this has led to regional investment bias $[6,7]$. In this study, we aim to illustrate that the combined risk of climate change and uncertain investment could further exacerbated future regional disparity.

Hazards such as flooding represent a significant challenge to urban planners and decision-makers, given the limited financial resources to protect transport networks for present and future adverse weather events. Currently, there are no tools to aid the prioritisation of investment in transport resilience, and understanding the relation between network exposure, demand and vulnerability is one of the priorities of the resilience research agenda [8]. In the UK, appraisal of transport schemes combines cost-benefit analysis within a broader multi-criteria analysis [9], and the centralised process of prioritising funding is facilitated by Benefit-Cost Ratio (BCR) methods, in which the costs of intervention are weighted against the benefits of that intervention to give a ratio score. The approach, which is rigorous and replicable, relies on the ability to quantify benefits as economic values and is based on a selection of costs and benefits. The Value of (travel) Time (VoT) is one of the major contributing factors to the BCR score [10-12], as it is functional for calculating users' benefits based on the value of their travelling time (per minute). Some have argued this has resulted in a heavy bias towards highway investment [13]. Thus, investment is more successful in schemes that promote the reduction in journey times (in the locality assessed), and in regions with higher travel demand, more transport users can generate more scheme benefits [14, 15]. Researchers have tried to value impacts of disruption on transport by utilising standard appraisal techniques [16, 17, 19-21]. The approaches have generally resulted in low estimated costs in comparison to the large investment costs of adaptations (e.g. new defences, network realignment, etc.), and this leaves regions at risk from complete severance from the network in some UK cases, such as the southwest rail network [20].

In light of this, and with the widespread recognition that climate change and flooding will increase the vulnerability of our transport systems, two important questions are raised: $(i)$ on a national scale, what is the regional disparity between risk of flooding to transport networks, and thus the need for future investment in adaptation schemes? (ii) to what extent is this coupled with the potential constraints (or bias) embedded within the BCR approach to funding allocation? The study aims to provide evidence and context to raise awareness of the future constraints regions may face, supporting an argument for a more evidenced-based system for valuing resilience in infrastructure investment. In the next section, we present the methodological framework and data used to answer the research questions (Section 2), we then present the results of the study (Section 3), and finally we discuss the results (Section 4) and implications for future research and regional developments.

\section{METHODOLOGY}

A framework is developed to answer the research questions raised in sec. 1 (see Fig. 1). As noted, adverse weather events have increased in frequency and/or magnitude, similarly populations and assets at risk have also increased. Such complex dynamics are likely to encompass negative consequences for disaster risk. The concept of risk includes three main elements: hazard, exposure and vulnerability; the interaction among these three elements determines the impact of adverse events [1]. 


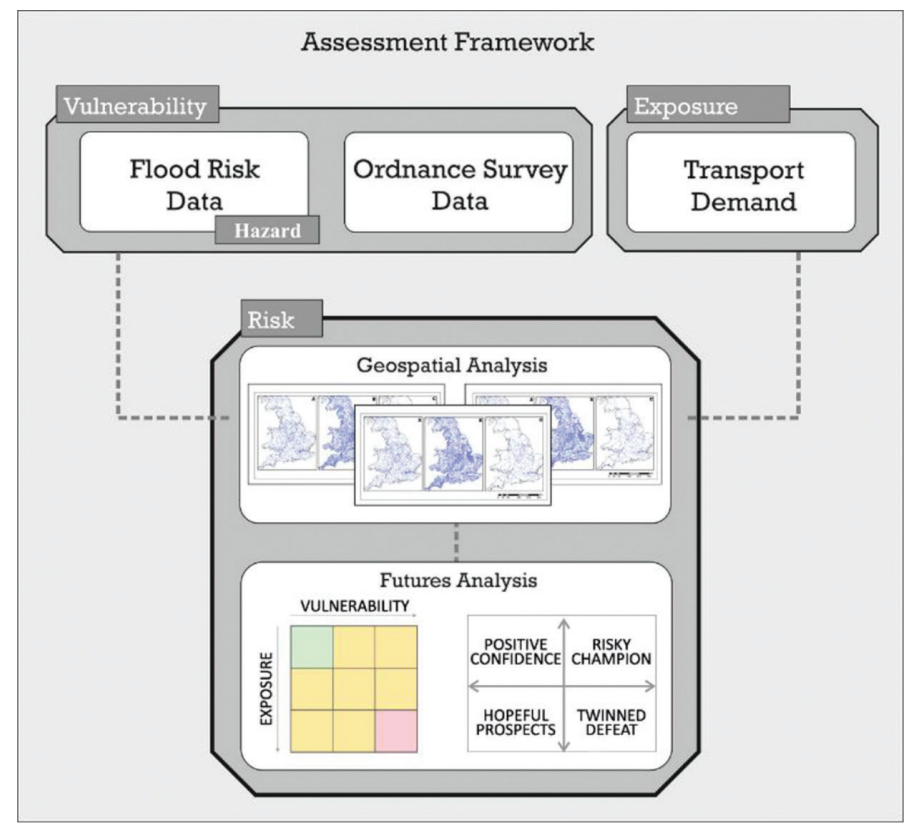

Figure 1: The methodological framework, following the concept of risk as defined by [1].

This paper is focused on identifying flooding risk at regional scale, as a basis for future study on adaptation and resilience. In this specific study, coastal and riverine flooding represents the hazard; the spatial location of road and railway networks, intercepted by the hazard maps, represents the vulnerability; and the demand (passenger use of the network) represents the exposure (and by extent, future investment potential). Figure 1 illustrates the methodological approach and further details now follow.

\subsection{Data and approach}

The flood risk of infrastructure assets is a well-established field of research, and there is growing interest on understanding the vulnerability of transport infrastructure [2]. However, in the UK, examples at national scales are rare (e.g. [22]), especially those that include a quantified analysis. We quantify regional risk to flooding, by evaluating the potential network vulnerability and demand for a specific flood scenario (i.e. including both low and high risk areas). The data includes both coastal and fluvial flood hazard, while pluvial flood risk is out of the scope of this paper at present. It is important to note that the data does not include a specific time horizon or the frequency/magnitude of events; it provides only provisional spatial indication of the potential flood risk based on geological records [23]. Due to the exploratory nature of the paper, and the scope of analysis required to assess national infrastructure on various temporal scales, this assumption is demined justifiable at present.

Geological hazard due to flooding [23] is intersected with UK transport network dataset [24] to identify the percentage of the network at risk. Such risk can be mapped and visualised though geospatial analysis [25], as Fig. 2 shows. In order to present the data effectively, the framework (Fig. 1) will allow the analysis of regional data to, firstly, establish the differences 


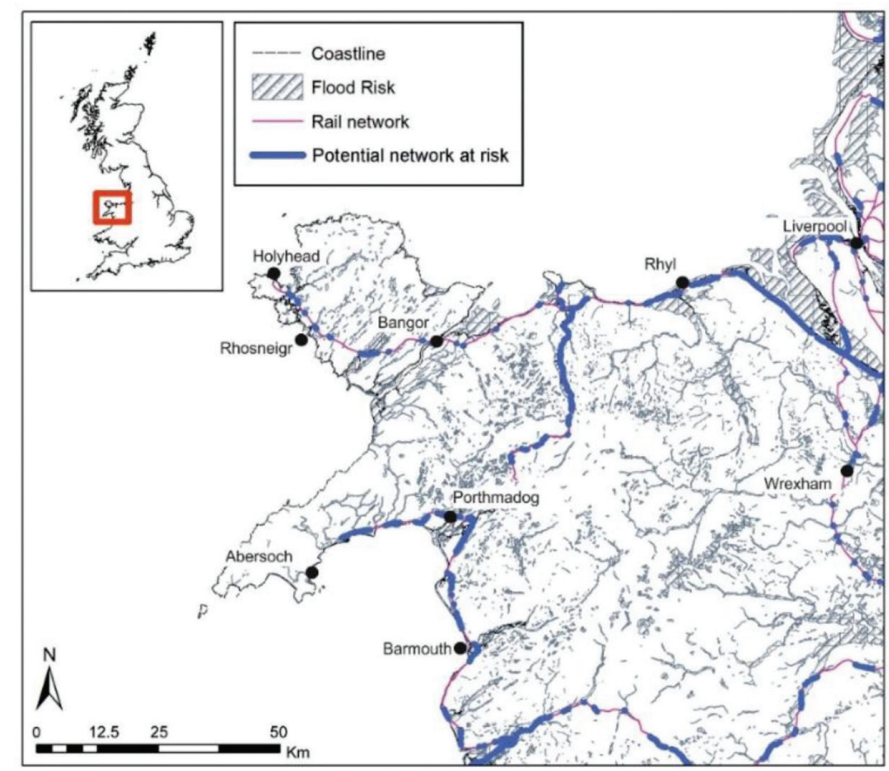

Figure 2: An example of identification of rail network risk for a region in the West of England by means of geospatial analysis. (Data source: British Geological Society, 2016; Ordnance Survey, 2017.)

in regional risk of flooding and, secondly, to examine which regions may be constrained by investment bias inherent in BCR approaches. The approach is visually and analytically enhanced by risk matrixes (Section 2.2) and quadrant matrixes (Section 2.3). These allow the investigation of total network passenger journeys to represent the fundamental exposure of regional transport services/functions to flooding. In addition, the data is used to represent the potential demand-based investment bias, assuming demand is a primary quantitative driver for investment in transport schemes and resilience improvements (this point is examined in further detail in Section 3).

\subsection{Qualitative risk analysis (risk matrix)}

Risk matrixes are one of the most widespread tool for qualitative risk analysis and assessment, since they help to clearly identify areas at risk [21, 26]. The risk matrix approach is used to examine the vulnerability and exposure of regional transport networks, and to identify low (L), medium (M) and high (H) risk areas (relative to one another), as shown in Fig. 3.

The three areas represent:

- Low risk (green): the risk is not as critical, because both exposure and vulnerability are at a low level. No action are usually recommended with this.

- Medium risk (yellow): it indicates areas to be monitored, since exposure or vulnerability are high and this can be linked to potential critical level of risk.

- High risk (red): high exposure and high vulnerability, it indicates an area in need of intervention since it has a critical level of risk. 


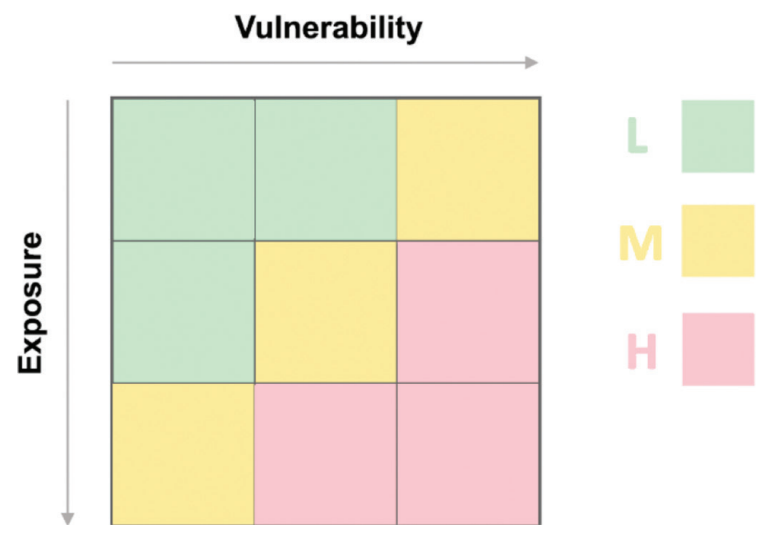

Figure 3: An example of risk matrix; green represents a lower risk, whereas red represents a higher risk (from [21]).

It must be stated that, on one hand, a risk matrix has the advantage to be very a practical and easy-to-visualise tool. It is also functional for promoting discussions based on complex data, in order to focus on the highest priority risk spots. On the other hand, a risk matrix by itself does not represent a robust decision-making tool. It is a qualitative-based tool that gives a preliminary risk ranking and more sophisticated calculations or tools are recommended for actual decision-making.

\subsection{Examining regional resilience futures (quadrant matrix)}

In order to integrate the analysis given by the risk matrixes and to examine which regions may be potentially constrained by investment bias inherent in the BCR approach, a quadrant matrix is used to generate future potential futures/scenarios (see Fig. 4). This type of

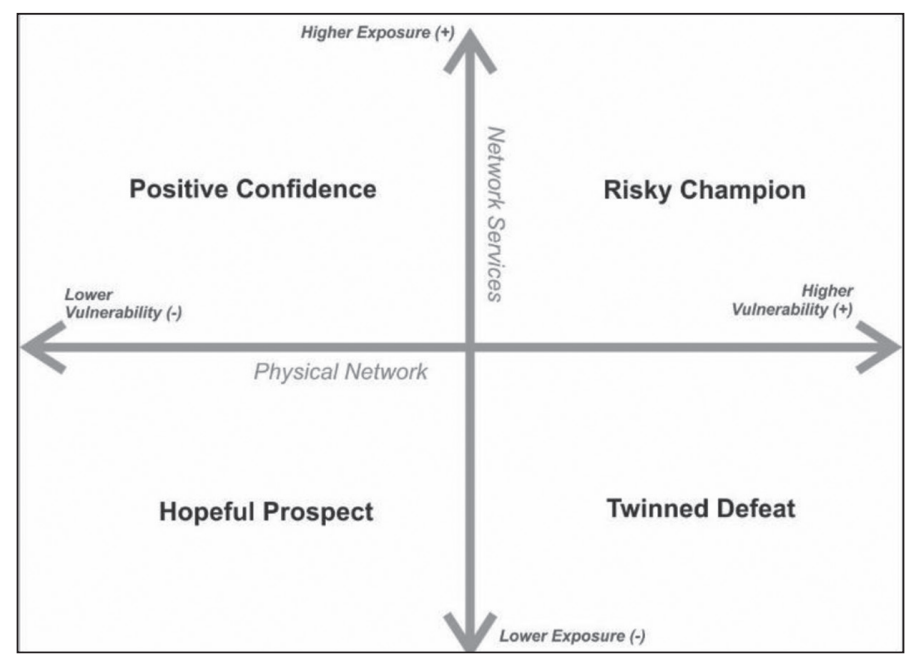

Figure 4: Framework for categorising network vulnerability on the basis of network exposure and vulnerability. 
approach is commonly used for the assessments of socio-economic futures and has been argued as an important aspect of transport vulnerability studies [3]. Scenario-based approaches provide a starting point for discussing potential outcomes of sector-based storylines regarding the impact of the future socio-economic environment on the transport sector. This approach is adapted to aid the narrative and categorisation of regions into potential futures based on: $i$ ) vulnerability, a relative quantification of network (length) in known flood risk areas (e.g. more than average or less than average risk), and ii) exposure, a relative quantification of daily travel use across modes (e.g. more than average or less than average demand), indicating the potential for generating more 'user benefits' for future adaptation schemes. The threshold for which indicators cross and thus actually categorise regions into one of the four squares ( $\mathrm{x}-\mathrm{y}$ axis crossing) is based initially on the average values from each data set.

The result of this approach provides four futures (Fig. 4) that each region could be associated with by:

- Positive Confidence: refer to regions with lower vulnerability to future flood risk and higher than average network demand exposure (e.g. positive investment bias).

- Risky Champion: refers to regions with higher vulnerability to flood risks and higher than average network demand exposure.

- Twinned Defeat: refers to regions with higher than average vulnerability to flood risk, and lower than average transport demand exposure (e.g. a potentially negative investment bias)

- Hopeful Prospect: refers to regions with lower vulnerability to flood risk and lower than average transport demand exposure.

This categorisation of regions only considers the most basic (high level) information on the transport systems and provides no attempt to examine individual or specific impacts of the risk exposure based on strategic importance (be that economic or social or both), and more complex factors of investment decisions (such as political, emergency response). The low level of complexity involved in this initial analysis is justified in order to provide impetus for progressive debate and research.

Risk matrices are considered approximate tools for estimating risk, but functional for qualitatively identifying critical priorities and least urgent situations [27]. The quadrant matrices can integrate the risk matrices analysis by interpreting the data from a point of view of scenarios and trends. Both methods are qualitative, but when associated they give a critical overview functional for decision-making.

The next section presents the results from the assessment exercise.

\section{RESULTS}

By examining the number of journeys on the road network in 2010-2016, Scotland, Wales, South West, Yorkshire and Humber, and the North East fall below the average national demand (journeys per day) across the network. Regions that have higher than national average demand include London, South East, and Eastern, however, modal differences between regions occur highlight the demand of particular networks (i.e. A roads or motorways).

From the geospatial analysis of the flood risk maps and regional transport networks, it can be seen that all roads in the UK are vulnerable to some flood risk, and our aggregated estimates assume between 10-30\% (average 15\%; this does not include local drainage surface flooding). Similarly, the UK rail network has between 13-36\% of its asset at risk from fluvial or coastal flooding (average $25 \%$ ). In terms of modes, the rail network is predominately more 
vulnerable to coastal and fluvial flooding and the regions of Wales, East Midlands, and Eastern have the highest proportion of networks in flood risk areas $(>30 \%)$. On the road network, London has the highest A road and motorway network in flood risk areas (30\% each), followed by the East Midlands (A roads) and the South West (motorways).

Figure 5 presents the results of the risk matrix analysis. "Low" data fell below the 25 th percentile, "medium" between the 25th and the 75th percentile, and "high" over the 75th percentile. South East and London are at the highest risk for both A roads and motorways, and railway. Eastern regions are critical for A roads and rail networks, while East Midland and South West are at high risk for A roads and motorways respectively.

The North East and the North West is non-critical for all the sectors; the South West and West Midlands are at low risk for A roads and railway. Scotland has a low level of risk for the road networks (both A roads and motorway), while the Scotland results non-critical for A roads and railway. Wales and Yorkshire and the Humber are associated to a potentially critical level of risk for both the road system (A road and motorways) and rail system.

At the final stage, the quadrant matrix approach is applied to the regional data to provide insight into potential futures for regional transport resilience (Fig. 6a and b). For UK motorways, Wales and the South West are considered to be at risk from the Twinned Defeat future,

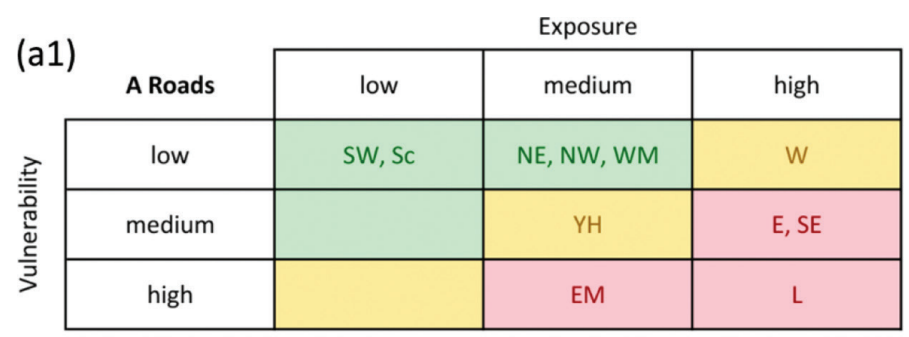

\begin{tabular}{|c|c|c|c|}
\hline \multirow[b]{2}{*}{ Motorways } & \multicolumn{3}{|c|}{ Exposure } \\
\hline & low & medium & high \\
\hline low & NE & $\mathrm{NW}, \mathrm{Sc}$ & W \\
\hline medium & & WM, YH, EM & SE \\
\hline high & $\mathrm{E}$ & SW & L \\
\hline
\end{tabular}

\begin{tabular}{|c|c|c|c|}
\hline \multirow[b]{2}{*}{ Rail } & \multicolumn{3}{|c|}{ Exposure } \\
\hline & low & medium & high \\
\hline low & & NW & \\
\hline medium & NE, WM, SW, & Sc & SE \\
\hline high & EM, W & E & L \\
\hline
\end{tabular}

Figure 5: Risk matrix analysis for: the main road system, (a1) A roads and (a2) Motorways; (b) the rail system in the UK. Abbreviations: East Midlands (EM), Eastern (E), London (L), North East (NE), North West (NW), Scotland (Sc), South East (SE), South West (SW), Yorkshire and the Humber (Y\&H), Wales (W), West Midlands (WM). 


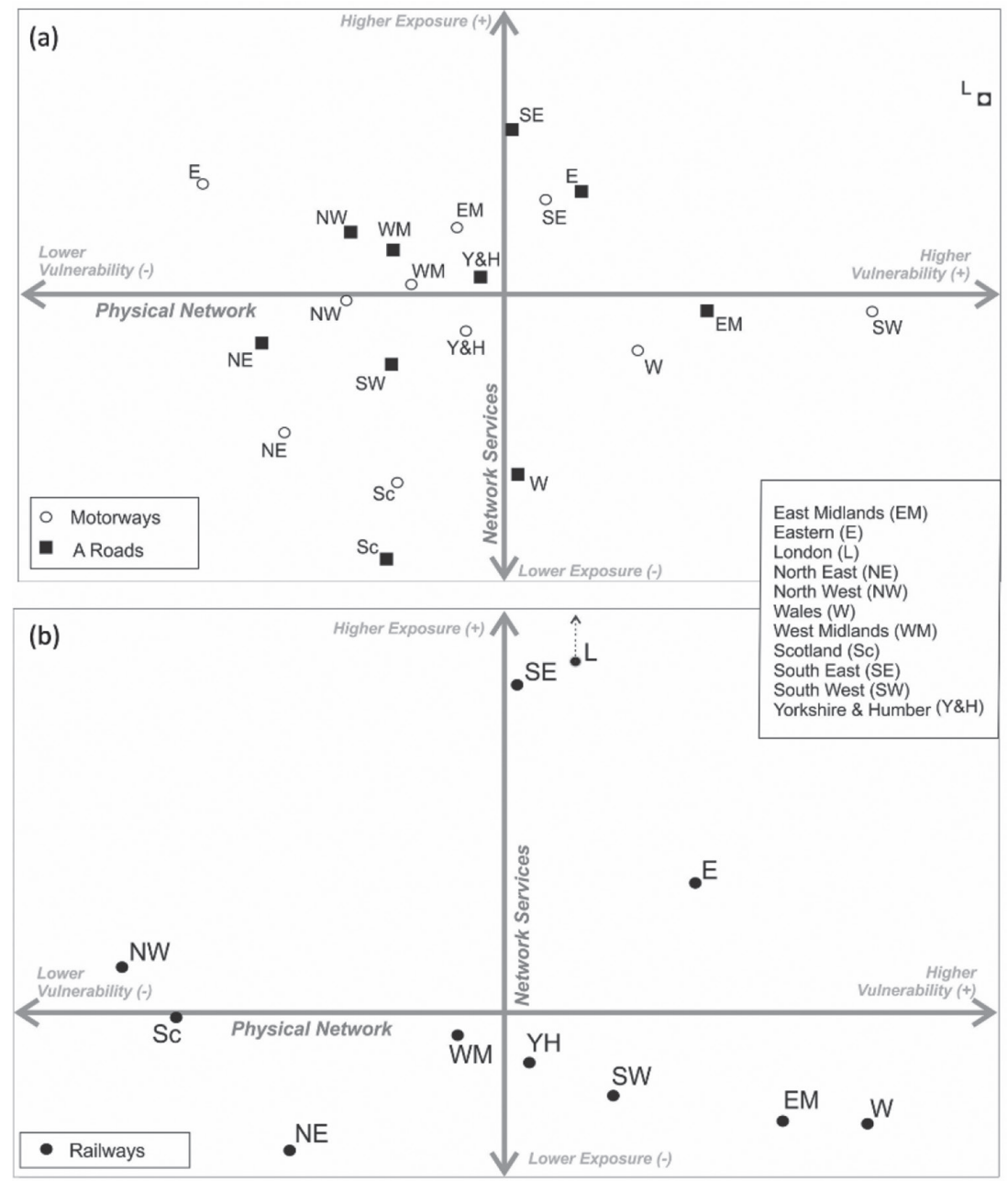

Figure 6: Regional transport futures based on vulnerability to flooding (network) and exposure (passenger demand) linked to investment potential (see Fig. 7) for (a) Road networks, and (b) rail networks. Note: the London rail network provides nearly $50 \%$ of all UK rail journeys and is only placed by its vulnerability to flooding.

along with East Midland and Welsh A Roads. The Risky Champion future is dominated by southern regions (London, South East, and Eastern), whilst many of the northern regions are situated in either Hopeful Prospects or Positive Confidence futures. Regarding the rail network, the extreme positive and negative locations were confirmed as North West (Positive Confidence), whilst Yorkshire and Humber, South West, Wales, and East Midlands are all at risk of Twinned Defeat futures.

It is interesting to examine the data behind the argument being raised regarding bias in investment in transport infrastructure. Figures $7 \mathrm{a}$ and $\mathrm{b}$ presents the regional data for construction spend against corresponding data for transport demand, providing preliminary evidence of the potential bias of investment spend on transport infrastructure. The level of 

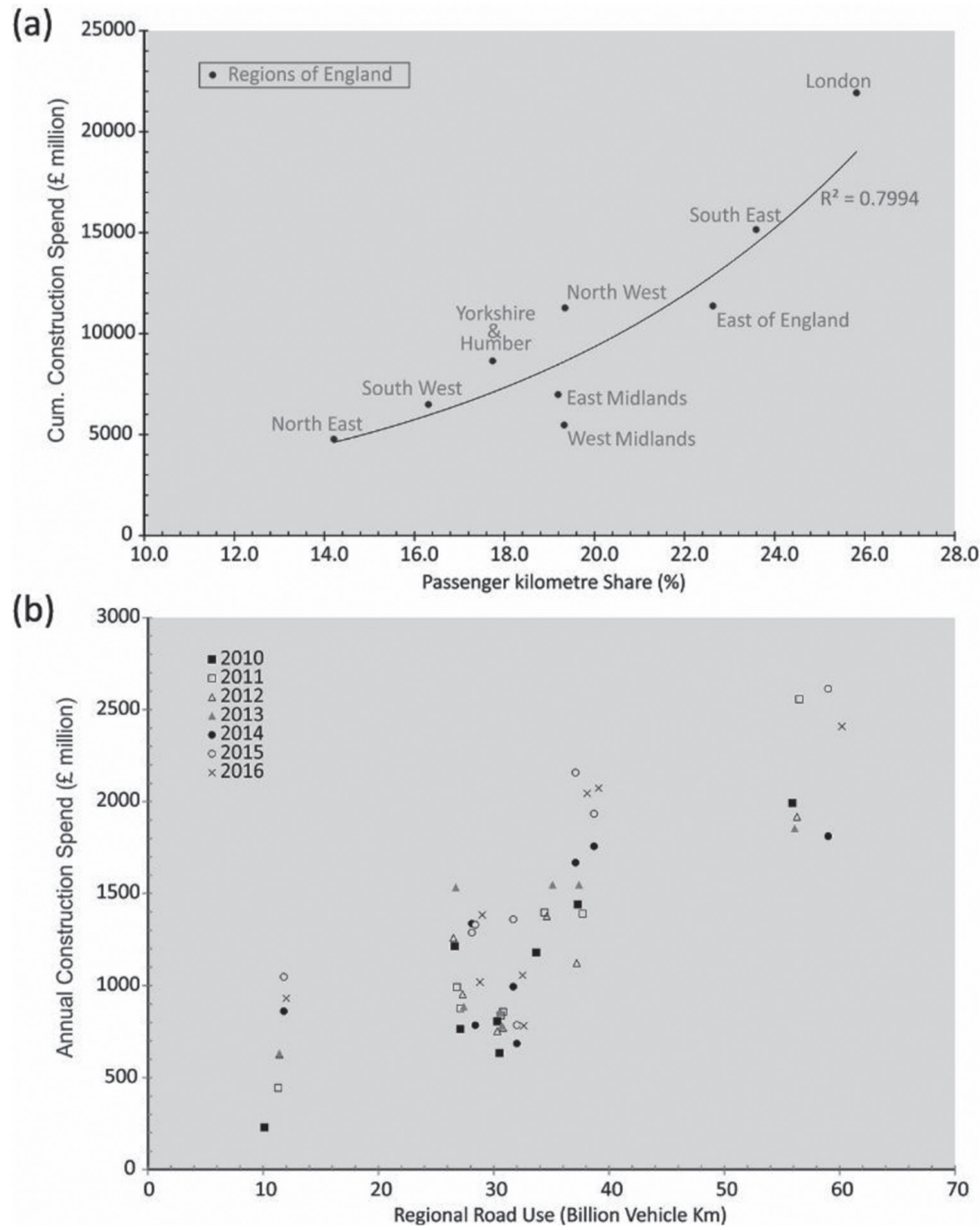

Figure 7: (a) Cumulative construction spend (2010-2016) against regional passenger kilometre share (2016) across the national strategic road network (A roads and motorways). (b) Annual construction spend against regional motor vehicle traffic (in vehicle Km) 2010-2016. Scotland and Wales are not included in this analysis. (Source: ONS, 2017.)

new infrastructure spend in the UK ( $80 \%$ of which is on transport infrastructure) appears to be disproportionately correlated to the volume of journeys within the UK network (Fig. 7a). For instance, the region with the lowest journey demand, North East, ( 15\% of the UK total), received almost the $80 \%$ less cumulative investment spend than the region with the highest journey demand (26\% of all journeys).

With the exception of the South West, there is a distinct North-South divide in investment. Observing annual construction spend and road use (vehicle $\mathrm{km}$ ) there are distinct clustering of regions that crudely follow a similar trend (Fig. 7b); the region with the lowest demand 
received almost $95 \%$ less annual funding than the region with the highest. It can also be seen that the most investment spend was received in 2015, whilst the least investment spend was received in 2010 .

In respect to this study, this provides some initial evidence of the potential risk of increased disparity in regions with a Twinned Defeat future (see Fig. 6), as they contain more kilometres of transport network at risk from flooding and will be potentially constrained by a lack of demand to help justify adaptation investments.

\section{DISCUSSION}

In this study, an initial framework was developed to investigate the future risks to transport infrastructure from flooding, by (i) examining the potential exposure to flood hazard; (ii) assessing regional patterns of flood risk; and (iii) speculating which regions may also be impacted and constrained by the current investment bias embedded within the BCR (Benefit Cost Ratio) approach to funding allocation. The findings provide a preliminary screening tool to assess the regional patterns of flood risk in the UK. Southern regions, for example, have higher risk of flooding than Northern regions. How all the regions build their case to justify investment in resilience remains a key question from both a methodological and a decision-making perspective. Yet Southern regions in the UK may benefit from their high journey demands to justify increased investment (Risky Champions), whilst the peripheral regions of Wales and the South West could face increasingly disrupted networks and will not benefit from higher demand benefits in their scheme appraisals. Assuming the Twinned Defeat scenario, the long-term effect of continued and prolonged disruption to their transport networks could impact regional competitiveness and GDP. It could be argued that Risky Champions should be prioritised due to their high level of service to the economy; however, this may well be at the deficit of other regions (e.g. Twinned Defeat/Hopeful Prospect). Thus, developing a tool to fairly and strategically prioritise long-term investments for transport infrastructure networks is a critical requirement for future regional equality.

This highly multi-disciplinary research (network modelling, climate science, economics) is characterised by a low-complexity analysis at this stage and the methodology developed could be improved in multiple ways. From a network risk perspective, the inclusion of surface water flooding and more dynamic (temporal and spatial) data is a logical progression. Stronger integration between the risk and quadrant matrix and primary indicators used can be made in order to help drive decision-making upon risk-based information. The growing availability of 'Big Data' could also help improve the robustness of the approach regarding maintenance [and operation regimes] and [potential] flooding (e.g. physical damage costs and disruption to service functions) thus building a more complete business case for examining future infrastructure investments. Assuming all future investment decisions will be made based on simply on BCRs is a major simplification of this study, and integrating well established decision making approaches (e.g. decision pathways) will be an important addition if the approach is to be taken up and utilised in strategic planning.

Finally, if adaptation funding is continually constrained, the question of how to respond to these future risks becomes even more prevalent. The role of transport users and more sustainable engineering techniques with increased non-monetary benefits will be important areas of study. The valuation models used for both transport schemes and flood schemes need to be carefully considered, and addressing the challenges raised in applying them in the transport resilience context requires strong interdisciplinary collaboration. It is hoped this study will provide impetus for further discussion and research to help improve long-term decision making for resilient transport systems. 


\section{CONCLUSION}

This study aimed to raise awareness around the level of risk of national infrastructure, alongside the allocated funding for improving its resilience, and make a step toward developing a tool to help prioritise investment in transport resilience beyond the piecemeal funding that dominates the sector currently. The results highlight current issues of existing trends and open the agenda around how to manage the transport system to future flood risk, in particular in the light of more impactful events. Identifying areas in need for extra attention is fundamental for evaluating a portfolio of alternative solutions and consequent investment, since flood risk could be magnified in the future. A preliminary methodology was developed to estimate portions of network at risk on the basis of their vulnerability and exposure, considering the strategic road network (A roads and motorways) and the railway network. Regional risk matrixes indicated a North/South divide in terms of current flood risk, whilst quadrant matrices examining the potential futures. The future categorisation resulted in contrast with current investment, which showed a disproportionate level of investment in networks with higher demand levels. With a non-complex methodology, the results provide strong evidence for the need to discuss appraisal approaches and their implication for national/regional infrastructure investment in climate adaptation and resilience. Ongoing studies will integrate the risk and quadrant matrix approaches more succinctly, along with developing the overall approach of a tool to help strategic planning and prioritisation of investment for long-term resilient infrastructure systems.

\section{ACKNOWLEDGEMENTS}

This work was supported by the Engineering and Physical Sciences Research Council (ESPRC) (EP/N017064/1), and the Leverhulme Trust (ECF-2014-177).

\section{REFERENCES}

[1] IPCC, Managing the Risks of Extreme Events and Disasters to Advance Climate Change Adaptation. A Special Report of Working Groups I and II of the Intergovernmental Panel on Climate Change [Field, C.B., V. Barros, T.F. Stocker, D. Qin, D.J. Dokken, K.L. Ebi, M.D. Mastrandrea, K.J. Mach, G.-K. Plattner, S.K. Allen, M. Tignor, and P.M. Midgley (eds.)]. Cambridge University Press, Cambridge, UK, and New York, NY, USA, 582 pp, 2012.

[2] Mattsson, L.-G. \& Jenelius, E., Vulnerability and resilience of transport systems - A discussion of recent research. Transportation Research Part A: Policy and Practice, 81, pp. 16-34, 2015. https://doi.org/10.1016/j.tra.2015.06.002

[3] Jaroszweski, D., Chapman, L. \& Petts, J., Assessing the potential impact of climate change on transportation: the need for an interdisciplinary approach. Journal of Transport Geography, 18(2), pp. 331-335, 2010. https://doi.org/10.1016/j.jtrangeo.2009.07.005

[4] Eddington, R., The Eddington Transport Study: Transport's role in sustaining the UK's productivity and competitiveness, HM Treasury, London, 2006.

[5] Banister, D. \& Berechman, J., Transport Investment and Economic Development, University College London Press, London, 2000.

[6] SPERI, Public infrastructure investment and business activity in the English regions (British Political Economy Brief No. 15). Sheffield Political Economy Research Institute (SPERI), 2015.

[7] IPPR, New transport figures reveal London gets $£ 1,500$ per head more than the North - but North West powerhouse 'catching-up', Institute for Public Policy Research, 2017 
[8] Birkmann, J. \& Mechler, R., Advancing climate adaptation and risk management. New insights, concepts and approaches: what have we learned from the SREX and the AR5 processes? Climatic Change, 133(1), pp. 1-6, 2015.

https://doi.org/10.1007/s10584-015-1515-y

[9] Department for Transport, Transport Analysis Guidance (TAG) Data Book, July 2017, $2017 \mathrm{a}$.

[10] Department for Transport, Transport Analysis Guidance (TAG) on the analysis of user and provider impacts in transport appraisals. TAG unit A1.3 user and provider impacts, March 2017, pp. 1-35, 2017b.

[11] Meunier, D. \& Quinet, E., Value of time estimations in cost benefit analysis: the French experience. Transportation Research Procedia, 8, pp. 62-71, 2015. https://doi.org/10.1016/j.trpro.2015.06.042

[12] Börjesson, M. \& Eliasson, J., Experiences from the Swedish value of time study. Transportation Research part A: Policy and Practice, 59(2014), pp. 144-158, 2014. https://doi.org/10.1016/j.tra.2013.10.022

[13] Hickman, R. \& Dean, M., Incomplete cost - incomplete benefit analysis in transport appraisal. Transport Reviews, pp. 1-21, 2017. https://doi.org/10.1080/01441647.2017.1407377

[14] Penning-Rowsell, E.C., Priest, S., Parker, D., Morris, J., Tunstall, S., Viavattene, C., Chatterton, J. \& Owen, D., Flood and coastal erosion risk management: A Manual for Economic Appraisal. Routledge, Middlesex (UK), 2013.

[15] Metroeconomica, Costing the impacts of climate change in the UK: Overview of guidelines. UKCIP Technical Report. UKCIP, Oxford, 2004.

[16] Dawson, D.A., Shaw, J. \& Gehrels, W.R, Sea-level rise impacts on transport infrastructure: The notorious case of the coastal railway line at Dawlish, England. Journal of Transport Geography, 51, pp. 97-109, 2016. https://doi.org/10.1016/j.jtrangeo.2015.11.009

[17] Pregnolato, M., Ford, A., Glenis, V., Wilkinson, S., \& Dawson, R., Impact of flooding and urban adaptation in a changing climate. Journal of Infrastructure Systems, 23(4), pp. 1-13, 2017.

[18] Pant, R., Hall, J. W. \& Blainey, S. P., Vulnerability assessment framework for interdependent critical infrastructures: case-study for Great Britain's rail network. European Journal of Transport and Infrastructure Research, 16(1), pp. 174-194, 2016

[19] British Geological Society, BGS indicators of flooding, 2016, available at http://www .bgs.ac.uk/products/hydrogeology/GIFMoreinfo.html (accessed 30th November 2017)

[20] Ordnance Survey, Integrated Transport Network (ITN) [GML2 geospatial data], Scale 1:1250, Tiles: GB, Updated: 22 May 2014, Ordnance Survey (GB), Using: EDINA Digimap Ordnance Survey Service, available at http://digimap.edina.ac.uk (accessed 30th November 2017).

[21] Jalayer, F., De Risi, R., De Paola, F., Giugni, M., Manfredi, G., Gasparini, P., Topa, M., Yonas, N., Yeshitela, K., Nebebe, A., Cavan, G., Lindley, S., Printz, A. \& Renner, F., Probabilistic GIS-based method for delineation of urban flooding risk hotspots. Natural Hazards, 73(2), pp. 975-1001, 2014.

https://doi.org/10.1007/s11069-014-1119-2

[22] CIRIA, Contaminated land risk assessment. A guide to good practice, CIRIA, London, 2001. 
[23] Larsen, M., Nielsen, N.H. \& Rasmussen, S.F., The blue spot model. Development of a screening to assess flood risk on national roads and highways system, Danish Road Directorate, Copenaghen (Denmark), 2010.

[24] Naso, S., Chen, A.S., Aronica, G.T. \& Djordjević, S., A novel approach to flood risk assessment: the Exposure-Vulnerability matrices. E3S Web Conf., 7, p. 08007, 2016.

[25] UKCIP, Socio-economic scenarios for climate change impact assessment: A guide to their use in the UK Climate Impacts Programme, UK Climate Impacts Programme, Oxford, 2001.

[26] ONS, Office of National Statistics. Developing new measures of infrastructure investment: July 2017, available at https://www.ons.gov.uk/ (accessed 30th November 2017).

[27] Elmontsri, M., Review of the strengths and weaknesses of risk matrices. Journal of Risk Analysis and Crisis Response, 4(1), pp. 49-57, 2014. https://doi.org/10.2991/ jrarc.2014.4.1.6 